\title{
Parametric analysis of entropy generation in magneto-hemodynamic flow in a semi-porous channel with OHAM and DTM ${ }^{1}$
}

\author{
M.M. Rashidi ${ }^{\mathrm{a}, *}$, A. Basiri Parsa ${ }^{\mathrm{b}}$, O. Anwar Bég ${ }^{\mathrm{c}, *}$, L. Shamekhi $^{\mathrm{b}}$, S.M. Sadrid ${ }^{\mathrm{d}}$ and Tasveer A. Bég \\ ${ }^{a}$ Mechanical Engineering Department, Engineering Faculty of Bu-Ali Sina University, Hamedan, Iran \\ ${ }^{\mathrm{b}}$ Young Researchers and Elite Club, Hamedan Branch, Islamic Azad University, Hamedan, Iran \\ ${ }^{\mathrm{c}}$ Department of Engineering and Mathematics, Sheffield Hallam University, Sheffield, England, UK \\ ${ }^{\mathrm{d}}$ Mechanical Engineering Department, Engineering Faculty of Bu-Ali Sina University, Hamedan, Iran \\ ${ }^{\mathrm{e}}$ Bio-Engineering Mechanics Research, Bradford, England, UK
}

\begin{abstract}
The magneto-hemodynamic laminar viscous flow of a conducting physiological fluid in a semi-porous channel under a transverse magnetic field has been analyzed by the optimal Homotopy Analysis Method (OHAM) and Differential Transform Method (DTM) under physically realistic boundary conditions first. Then as the main purpose of this study the important designing subject, entropy generation of this system, has been analyzed. The influence of Hartmann number (Ha) and transpiration Reynolds number (mass transfer parameter, Re) on the fluid velocity profiles in the channel are studied in detail first. After finding the fluid velocity profiles, graphical results are presented to investigate effects of the Reynolds number, Hartmann number, $x$-velocity of the moving plate, suspension height and dimensionless horizontal coordinate on the entropy generation.
\end{abstract}

Keywords: Entropy generation, optimal homotopy analysis method (OHAM), differential transform method (DTM), magnetohemodynamics, blood flow control, nonlinear differential equations

\footnotetext{
${ }^{1}$ Dedicated respectfully to Professor T.K. Hung, Professor of Bioengineering, Swanson School of Engineering, University of Pittsburgh, USA, on the occasion of his 75th birthday (2011), in recognition of his seminal achievements in biomechanics research and education and his benevolent support to the authors in their studies of biofluid dynamics.

${ }^{*}$ Corresponding author: M.M. Rashidi, Professor of Mechanical Engineering, Mechanical Engineering Department, Engineering Faculty of Bu-Ali Sina University, Hamedan, Iran. E-mail: mm_rashidi@yahoo.com and O. Anwar Bég, Director, Biomechanics and Biotechnology Research, Aerospace Engineering, Department of Engineering and Mathematics, Sheaf Building, Sheffield Hallam University, Sheffield S1 1WB, UK, England. Tel.: +98 811 8257409; Fax: +98 811 8257400; E-mail: o.beg@ shu.ac.uk.
}

\section{Nomenclature}

$B \quad$ strength of magnetic field

c nonlinear operator of the HAM

DTM differential transform method

$\mathrm{Ha}$ Hartmann number

OHAM optimal Homotopy analysis method

$\mathcal{L} \quad$ linear operator of the HAM

$l \quad$ suspension height

$L_{x} \quad$ length of the moving plate

$N_{G} \quad$ entropy generation parameter

$P \quad$ pressure

$q \quad$ mass transfer parameter (wall transpiration velocity) 
Re Reynolds number

$U \quad$ dimensionless velocity in the $x$-direction

$u \quad$ dimensionless $x$-component velocity

$u_{-} 0 \quad x$-velocity of the $\mathrm{m}$

$u^{*} \quad$ velocity component in the $x$ direction

$V \quad$ dimensionless velocity in the $y$-direction

$v \quad$ dimensionless $y$-component velocity

$v^{*} \quad$ velocity component in the $y$ direction

$x$ dimensionless horizontal coordinate

$x^{*} \quad$ distance in the $x$-direction parallel to the plates

$y \quad$ dimensionless vertical coordinate

$y^{*} \quad$ distance in the $y$-direction parallel to the plates

\section{Greek symbols}

$\rho$ biofluid density

$v \quad$ kinematic viscosity of biofluid

$\sigma \quad$ electrical conductivity of biofluid

$\varepsilon \quad$ aspect ratio $l / L_{x}$

\section{Introduction}

Boundary-layer problem is one of the problems that are described through nonlinear differential equations. In most cases, nonlinear problems do not have precise analytic solution. Boundary-layer flow and heat transfer of viscous fluids over a stretching surface occurs in many manufacturing processes, such as wire drawing, metal extrusion, drawing of copper wires, hot rolling, glass-fiber, glass blowing and metal spinning. Magneto-hydrodynamics (MHD) is the study of the interaction of conducting fluids with electromagnetic phenomena [1]. The flow of an electrically conducting fluid in the presence of a magnetic field is of importance in various areas of technology and engineering such as MHD power generation, MHD flow meters, MHD pumps, etc [2-4].

The conducting nature of blood has been confirmed to be generated by the presence of iron in the hemeoglobin molecule as described in [5-7] and also ionic content. The problem of control blood flow velocities in surgical procedures, magnetic drug targeting [8] and evaluating the influence of strong magnetic fields on the human circulation system. A number of analytical and computational studies of magnetohemodynamic flows have been communicated. The historical development of this subject was first presented by Bég [9] and Prasad et al. [10]. Keltner et al. [11] conducted one of the first significant analyses of magneto-hemodynamic flow, considering the pressure changes in vessels of the human vascular system. Sud and Sekhon [12] modeled the hydromagnetic blood flow via a rigid tube network using finite elements, showing that both the intensity and orientation of a magnetic field have substantial effects on flow profiles. Rao and Deshikachar [13] used a vorticity formulation to study oscillatory effects on hydromagnetic blood flow in variable cross-section channels, showing a distinct reduction in flow intensity with applied magnetic field. Mustapha et al. [14] studied axisymmetric magnetohydrodynamic blood flow through the arteries having irregular shaped multi-stenoses using the marker and cell (MAC) and successive-over-relaxation (SOR) numerical methods, aimed at evaluating magneto-therapy procedures. They identified that the flow separation region continues to diminish with the increasing intensity of the magnetic-field and vanishes for high Hartmann numbers. Newtonian viscous flows simulate accurately the transport in larger blood vessels [15]. Further Newtonian viscous hydromagnetic physiological flow studies have been communicated by Ramamurthy and Shanker [16] and Takhar et al. [17]. Pulsatile heat transfer in magnetic blood flow be studied by Bhargava et al [18]. Pandey and Tripathi [19] studied the hydromagnetic peristaltic flow in a conduit. Similar studies were reported by Mekheimer [20] and Pandey and Tripathi [21]. The numerical solution of magnetohydrodynamic (MHD) signal produced by moving conductive blood flow in the great vessels of the heart, under a static magnetic field done by Kainz et al. [22]. Das and Saha [23] studied magnetohydrodynamic pulsating blood flow in a rough thin-walled distensible conduit. A analysis of a dusty model for the axisymmetric flow of blood through coaxial tubes such that the outer tube with an axially nonsymmetreic has been done by Mekheimer and Kot [24]. Porous tubes by viscous non-conducting hemodynamic flow have also received much attention in recent years owing to applications in dialysis of blood in artificial kidneys [25-27]. An exact solution of the Navier-Stokes equations for steady two-dimensional laminar flow of a viscous, incompressible fluid in a channel with parallel, rigid, porous walls driven by uniform, steady suction (or injection) at the walls be presented by Berman [28]. More recently, Desseaux [29] extended 
Berman's study to analyze the influence of a magnetic field on laminar viscous flow in a semi-porous channel. Mekheimer et al. [30] studied the peristaltic transport of an incompressible viscous fluid due to an asymmetric waves propagating on the horizontal sidewalls of a rectangular duct under long-wavelength and low-Reynolds number assumptions.

Most scientific problems and phenomena such as Boundary-layer problem can convert to a nonlinear systems of ordinary or partial differential equations. In the present work we employ Berman's similarity transformation and study Berman-Desseaux flow i.e. magneto-hemodynamic flow in a semi-porous channel. This allows the reduction of the governing partial differential equations into a set of coupled nonlinear ordinary differential equations. We have difficulty usually in finding their exact analytical solutions. Explicit solutions to the nonlinear equations are of fundamental importance. Except a limited number of these problems that have precise analytical solution, most of them do not have analytical solution, so these nonlinear equations should be solved using other methods. In recent decades, much attempt has been done to the newly developed methods to introduce an analytic solution of these equations; one of these is perturbation method [31] that is strongly dependent upon the so-called "small parameters Thus, it is worthwhile developing some new analytic techniques independent of small parameters. In the other hand, the perturbation method cannot provide us with a simple way to adjust and control the convergence region and rate of given approximate series. Another one method is differential transform method that has been used in resent years frequently, such as [32, 33]. In 1992, Liao introduced the basic ideas of the homotopy in topology to propose a general analytic method for nonlinear problems, namely homotopy analysis method (HAM), [34] that does not need to any small parameter. This method has been successfully applied to solve many types of nonlinear problems by others [35-37]. Based on homotopy of topology, the validity of the HAM is independent of whether or not there exist small parameters in the considered equation. Therefore, the HAM can overcome the foregoing restrictions and limitations of perturbation methods [38]. This method also provides us with great freedom to select proper basic functions to approximate solutions of nonlinear problems. Using one interesting property of homotopy, one can transform any nonlinear problem into an infinite number of linear problems.
In addition, another powerful semi-numerical method, the differential transform method (DTM), which is based on Taylor series expansion. The concept of the DTM was first proposed by Zhou [39], who solved linear and nonlinear problems in electrical circuit problems. Chen and Ho [40] developed this method for partial differential equations and Ayaz [41] plied it to a system of differential equations. Jang et al. [42] presented the two-dimensional DTM for the solution of partial differential equations. This method has been successfully applied to various application problems. Other interesting studies employing DTM simulation include Kumaz et al. [43] and Rashidi et al. [44] for nanofluid heat and mass transfer. Basiri Parsa et al. [45] presented the seminumerical techniques known as OHAM and DTM for solution of the governing equations of present problem.

In recent years, many studies have been published on the entropy generation as an important thermodynamic factor in theoretical and industrial applications. Entropy generation analysis, which is a thermodynamic approach, is used to optimize the thermal engineering devices for higher energy efficiency [46]. The performance of engineering equipment in the presence of the irreversibilities is reduced and entropy generation is a measure of the level of the available irreversibilities in a process. Entropy generation can be used as a quantitative measure of irreversibilities that is associated with a process, because of this fact that; the greater the entropy generation indicates the greater the extent of irreversibilities.

Aiboud and Saouli [47] illustrated the application of the second law analysis of thermodynamics to viscoelastic magneto-hydrodynamic flow over a stretching surface analytically using Kummer's functions. Rashidi et al. have been derived the entropy generation equation for the steady MHD flow due to a rotating porous disk in a nanofluid [48]. Also the entropy generation in off-centered stagnation flow towards a rotating disc has been analyzed parametrically by Rashidi et al. [49].

In the present article, we review briefly the fundamentals of OHAM and the DTM and then apply both methods to solve the transformed coupled nonlinear ordinary differential equations for laminar magnetohemodynamic, viscous flow in a semi-porous channel. The main goal of the present study is to analyzing the entropy generation in this case. For this propose, results of OHAM and DTM are used. 


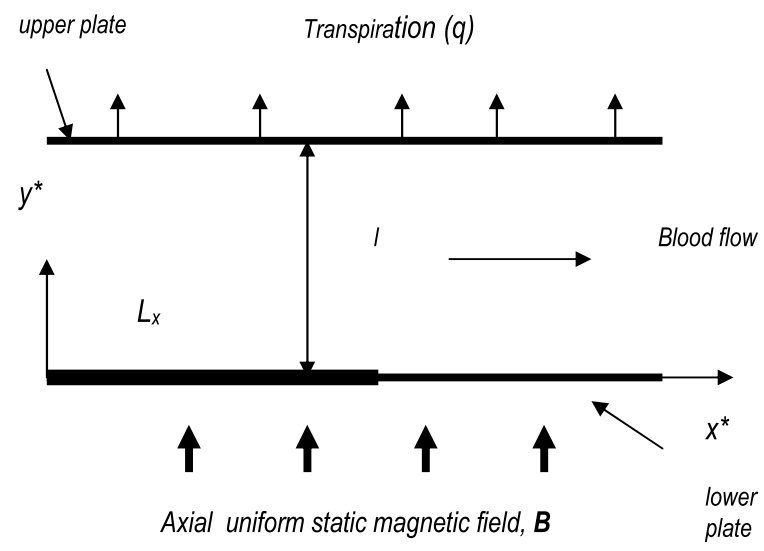

Fig. 1. Schematic diagram of the problem.

\section{Mathematical formulation}

Present problem is laminar two-dimensional stationary flow of an electrically conducting incompressible viscous fluid in a semi-porous channel consisting of a long rectangular plate of length $L_{x}$ in uniform translation in the $x^{*}$ direction and an infinitely long porous plate situated at a vertical distance 1 above the first plate. The distance between the two plates is $l$. The physical fluid properties are constant. Lateral mass flux is permitted i.e. a normal transpiration velocity, $\mathrm{q}$, is present at the porous plate. A uniform magnetic field $B$ is imposed in the $\mathrm{y}^{*}$-direction. The physical regime is shown in Fig. 1 [29].

In the case of a short circuit the electrical field and perturbations to the basic normal field can be neglected, and furthermore in the absence of gravity forces, the governing equations are as follows [50, 51]:

$$
\frac{\partial u^{*}}{\partial x^{*}}+\frac{\partial v^{*}}{\partial y^{*}}=0
$$

$$
\begin{gathered}
u^{*} \frac{\partial u^{*}}{\partial x^{*}}+v^{*} \frac{\partial u^{*}}{\partial y^{*}} \\
=-\frac{1}{\rho} \frac{\partial P^{*}}{\partial x^{*}}+v\left(\frac{\partial^{2} u^{*}}{\partial x^{* 2}}+\frac{\partial^{2} u^{*}}{\partial y^{* 2}}\right)-u^{*} \frac{\sigma B^{2}}{\rho}, \\
u^{*} \frac{\partial v^{*}}{\partial x^{*}}+v^{*} \frac{\partial v^{*}}{\partial y^{*}}=-\frac{1}{\rho} \frac{\partial P^{*}}{\partial y^{*}}+v\left(\frac{\partial^{2} v^{*}}{\partial x^{* 2}}+\frac{\partial^{2} v^{*}}{\partial y^{* 2}}\right)
\end{gathered}
$$

The appropriate boundary conditions for the velocity field are:

$$
y^{*}=0: u^{*}=u_{0}^{*}, v^{*}=0,
$$

$$
y^{*}=h: u^{*}=0, v^{*}=-q .
$$

The mean velocity $U$ is evaluated by means of the following relation:

$$
U \times h=\int_{0}^{h} u^{*} \times d y^{*}=L_{x} \times q .
$$

The following transformations:

$$
\begin{gathered}
x=\frac{x^{*}}{L_{x}}, y=\frac{y^{*}}{l}, \\
u=\frac{u^{*}}{U}, \quad v=\frac{v^{*}}{q}, \quad P_{y}=\frac{P^{*}}{\rho \cdot q^{2}} .
\end{gathered}
$$

Now the two key dimensionless hydrodynamic parameters namely the Hartmann number $\mathrm{Ha}$ (for magnetohydrodynamic body force effects) [29] and the transpiration Reynolds number, Re for the lateral mass flux and dynamic forces are defined:

$$
\begin{gathered}
H a=B l \sqrt{\frac{\sigma}{\rho . v}}, \\
\operatorname{Re}=\frac{l q}{v} .
\end{gathered}
$$

Substituting Equations (6-10) into Equations (1 and 3 ) leads to the dimensionless mass and momentum conservation equations:

$$
\frac{\partial u}{\partial x}+\frac{\partial v}{\partial y}=0
$$

$$
\begin{gathered}
u \frac{\partial u}{\partial x}+v \frac{\partial u}{\partial y} \\
=-\varepsilon^{2} \frac{\partial P_{y}}{\partial x}+\frac{v}{l q}\left(\varepsilon^{2} \frac{\partial^{2} u}{\partial x^{2}}+\frac{\partial^{2} u}{\partial y^{2}}\right)-u \frac{H a^{2}}{\operatorname{Re}}, \\
u \frac{\partial v}{\partial x}+v \frac{\partial v}{\partial y}=-\frac{\partial P_{y}}{\partial x}+\frac{v}{l q}\left(\varepsilon^{2} \frac{\partial^{2} v}{\partial x^{2}}+\frac{\partial^{2} v}{\partial y^{2}}\right) .
\end{gathered}
$$

In Equations (12 and 13), the quantity $\varepsilon$ is defined as the ratio of the distance $l$ and a characteristic length $L_{x}$ of the moving plate. This ratio is normally small [29]. Berman's similarity transformation is used to eliminate the aspect ratio $\varepsilon$ from the momentum Eqns,

$$
v=-V(y), u=\frac{u^{*}}{U}=u_{0} U(y)+x \frac{d V}{d y} .
$$

Substituting Equation (14) in the second momentum equation (13) shows that quantity $\partial P_{y} / \partial y$ does not 
depend on the longitudinal variable $x$. Inspection of the first momentum equation, also reveals that $\partial^{2} P_{y} / \partial x^{2}$ is independent of $x$. Separation of variables (omitting the asterisks) leads to

$$
\begin{aligned}
& V^{\prime 2}-V V^{\prime \prime}-\frac{1}{\operatorname{Re}} V^{\prime \prime \prime}+\frac{H a^{2}}{\operatorname{Re}} V^{\prime} \\
& =\varepsilon^{2} \frac{\partial^{2} P_{y}}{\partial x^{2}}=\varepsilon^{2} \frac{1}{x} \frac{\partial P_{y}}{\partial x}, \\
& U V^{\prime}-V U^{\prime}=\frac{1}{\operatorname{Re}}\left[U^{\prime \prime}-H a^{2} U\right] .
\end{aligned}
$$

The right-hand side of Equation (15) is constant. So, this equation with respect to $y$ can be derived.

$$
V^{I V}=H a^{2} V^{\prime \prime}+\operatorname{Re}\left[V^{\prime} V^{\prime \prime}-V V^{\prime \prime \prime}\right]
$$

where primes denote differentiation with respect to $y$ and asterisks have been omitted for simplicity. The velocity boundary conditions become:

$$
\begin{aligned}
& y=0: \quad U=1, \quad V=0, \quad V^{\prime}=0, \\
& y=1: \quad U=0, \quad V=1, \quad V^{\prime}=0 .
\end{aligned}
$$

\section{HAM and DTM solutions}

\subsection{HAM}

The basic ideas of HAM are documented in [34]. From Equations (17 and 16) and with boundary conditions (18), the initial approximations of $V(y)$ and $U(y)$ are chosen as $\mathrm{V}_{0}(\mathrm{y})=y-(1 / 2 \pi) \sin (2 \pi \mathrm{y}), U_{0}(y)=$ $1-y$. Selection criteria of initial approximations is satisfaction of boundary conditions, thus they are not unique. The linear operators are defined as:

$$
\begin{aligned}
& \mathcal{L}[V(y ; p)]=\frac{\partial^{4} V(y ; p)}{\partial t^{4}}, \\
& \mathcal{L}[U(y ; p)]=\frac{\partial^{2} U(y ; p)}{\partial t^{2}} .
\end{aligned}
$$

Furthermore, Equations (17 and 16) suggest the following definitions of the nonlinear operators:

$$
\begin{gathered}
\mathcal{N}[V(y ; p)]=\frac{\partial^{4} V(y ; p)}{\partial y^{4}}-H a^{2} \frac{\partial^{2} V(y ; p)}{\partial y^{2}} \\
-\operatorname{Re}\left(\frac{\partial V(y ; p)}{\partial y} \frac{\partial^{2} V(y ; p)}{\partial y^{2}}-V(y ; p) \frac{\partial^{3} V(y ; p)}{\partial y^{3}}\right),
\end{gathered}
$$

$$
\begin{gathered}
\mathcal{N}[U(y ; p)]=U(y ; p) \frac{\partial V(y ; p)}{\partial y}-V(y ; p) \frac{\partial U(y ; p)}{\partial y} \\
-\frac{1}{\operatorname{Re}}\left(\frac{\partial^{2} U(y ; p)}{\partial y}-H a^{2} U(y ; p)\right) .
\end{gathered}
$$

Using the above definition, with assumption auxiliary functions (see $\operatorname{Ref}[45]) H_{V}(y)=1, H_{U}(y)=1$, we construct the zero-order deformation equation:

$(1-p) \mathcal{L}\left[\varphi(x ; p)-u_{0}(x)\right]=H_{\varphi}(x) p \hbar \mathcal{N}[\varphi(x ; p)]$.

Differentiating the zeroth - order deformation equation (23) $m$ times with respect to $p$, and finally dividing by $m$ !, generates the $m$ th - order deformation equation:

$$
\begin{aligned}
& \mathcal{L}\left[V_{m}(y)-\chi_{\mathrm{m}} V_{m-1}(y)\right]=\hbar R_{m}\left(\vec{V}_{m-1}\right), \\
& \mathcal{L}\left[U_{m}(y)-\chi_{\mathrm{m}} U_{m-1}(y)\right]=\hbar R_{m}\left(\vec{U}_{m-1}\right),
\end{aligned}
$$

subject to the initial conditions:

$$
\begin{aligned}
& U_{m}=0, V_{m}=0, V_{m}^{\prime}=0 \text { at }: \quad y=0, \\
& U_{m}=0, V_{m}=0, \quad V_{m}^{\prime}=0 \text { at }: y=1,
\end{aligned}
$$

where

$$
\begin{aligned}
& R_{m}\left(\vec{V}_{m-1}\right) \\
& =\frac{\partial^{4} V_{m-1}(y)}{\partial y^{4}}-H a^{2} \frac{\partial^{2} V_{m-1}(y)}{\partial y^{2}} \\
& -\operatorname{Re}\left(\sum_{j=0}^{m-1} \frac{\partial V_{j}(y)}{\partial y} \frac{\partial^{2} V_{m-1-j}(y)}{\partial y^{2}}\right. \\
& \left.-\sum_{j=0}^{m-1} V_{j}(y) \frac{\partial^{3} V_{m-1-j}(y)}{\partial y^{3}}\right),
\end{aligned}
$$

$$
\begin{aligned}
& R_{m}\left(\vec{U}_{m-1}\right) \\
& =\sum_{j=0}^{m-1} U_{j}(y) \frac{\partial V_{m-1-j}(y)}{\partial y}-\sum_{j=0}^{m-1} V_{j}(y) \frac{\partial U_{m-1-j}(y)}{\partial y} \\
& \quad-\frac{1}{\operatorname{Re}}\left(\frac{\partial^{2} U_{m-1}(y)}{\partial y^{2}}-H a^{2} U_{m-1}(y)\right)
\end{aligned}
$$

The solution of the $m$ th - order deformation equations (24) and (25) for $m \geq 1$ can be done simply.

The final approximate solution can be obtained $V_{a p p}=\sum_{i=0}^{n} V_{i}, U_{a p p}=\sum_{i=0}^{n} U_{i}$. In general, by means of the so-called $\hbar$ - curve by Liao [34], the valid region of $\hbar$ is the horizontal line segment. To see the 


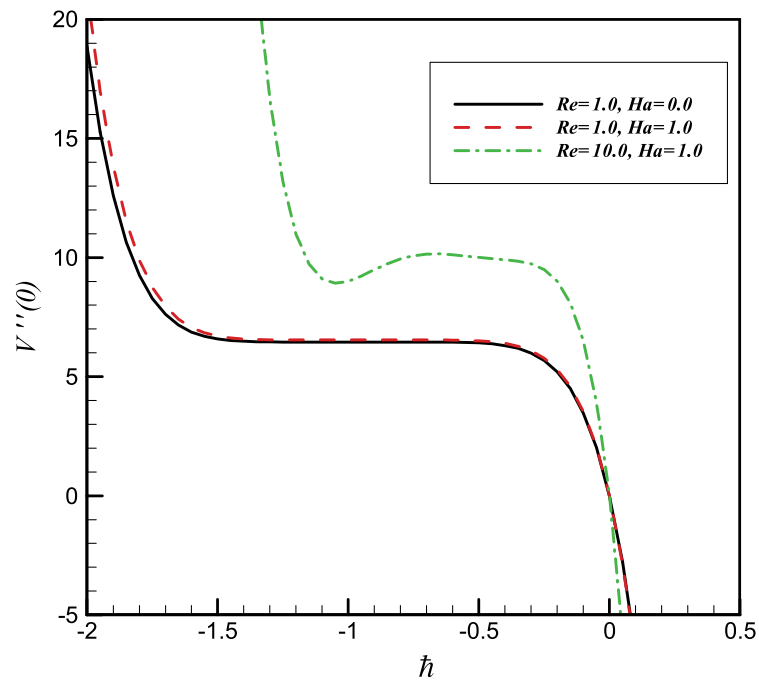

Fig. 2. The $\hbar$-curve of $V^{\prime \prime}(0)$ given by the 10 th-order approximate solution, for various values of Re and $\mathrm{Ha}$, [45].

range of admissible values of the auxiliary parameter $\hbar$, for various values of $\mathrm{Re}$ and $\mathrm{Ha}$, the curves of $\hbar$ are plotted in Figs. 2 and 3 associated with the 10thorder approximation. The optimal value of auxiliary parameter is obtained as follow:

$$
\begin{aligned}
& E_{V, m}=\frac{1}{K} \sum_{i=0}^{K}\left[\mathcal{N}_{V}\left(\sum_{i=0}^{m} V_{i}(i \Delta x)\right)\right]^{2}, \\
& E_{U, m}=\frac{1}{K} \sum_{i=0}^{K}\left[\mathcal{N}_{U}\left(\sum_{i=0}^{m} U_{i}(i \Delta x)\right)\right]^{2},
\end{aligned}
$$

where $\Delta x=10 / K$ and $K=20$. For a given order of approximatio, $m$, the optimal values $\hbar$ are given by the minimum of $E_{m}$, corresponding to the following nonlinear algebraic equations:

$$
\frac{d E_{V, m}}{d \hbar}=0, \quad \frac{d E_{U, m}}{d \hbar}=0 .
$$

Table 1 shows the optimal values obtained for the auxiliary parameter $\hbar$ [45]. To see the accuracy of the solutions, the residual errors are defined for the system as follows:

$$
\begin{aligned}
R E_{V}= & V_{n}^{I V}(y)-H a^{2} V^{\prime \prime}{ }_{n}(y)+\operatorname{Re}\left[V^{\prime}{ }_{n}(y) V_{n}^{\prime \prime}{ }_{n}(y)\right. \\
& \left.-V_{n}(y) V_{n}^{\prime \prime \prime}(y)\right]
\end{aligned}
$$

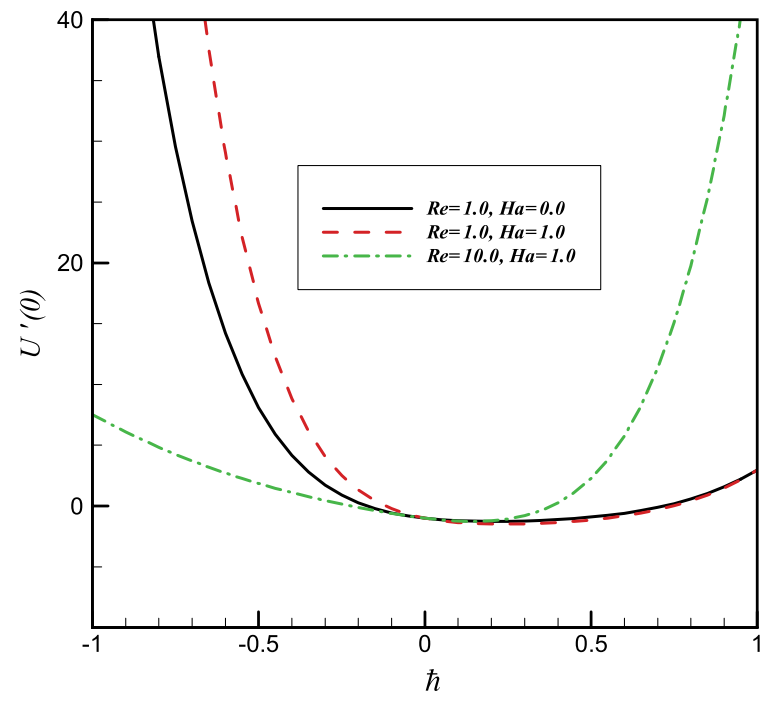

Fig. 3. The $\hbar$-curve of $U^{\prime}(0)$ given by the 10 th-order approximate solution, for various values of $\operatorname{Re}$ and $H a$, [45].

$$
\begin{aligned}
R E_{U}= & U_{n}(y) V_{n}^{\prime}(y)-V_{n}(y) U^{\prime}{ }_{n}(y) \\
& -\frac{1}{\operatorname{Re}}\left[U^{\prime \prime}{ }_{n}(y)-H a^{2} U_{n}(y)\right],
\end{aligned}
$$

where $V_{n}(y)$ and $U_{n}(y)$ are the HAM solutions for $V(y)$ and $U(y)$. Figures 4 and 5 show the residual error for the case when $\mathrm{Re}=1.0, \mathrm{Ha}=1.0$ given by 10 thorder approximation [45]. These figures show that a 10th-order approximation yields the best accuracy for the current problem. Also, Figs. 6-8 respectively, show $V(y), V^{\prime}(y)$ and $U(y)$ obtained by various order of the OHAM and numerical method (fourth-order Runge-Kutta quadrature with a shooting method) for a special case when $\operatorname{Re}=1.0, H a=1.0$, [45].

\subsection{DTM}

The transformation of the $k$ th derivative of a function $f$ in one variable is as follows [39]:

$$
F(k)=\frac{1}{k !}\left[\frac{d^{k} f(x)}{d x^{k}}\right]_{x=x_{0}},
$$

and the inverse transformation is defined by:

$$
f(x)=\sum_{k=0}^{\infty} F(k)\left(x-x_{0}\right)^{k} .
$$

The fundamental mathematical operations performed by the differential transform method (DTM) are listed in Ref [39]. Taking the differential transform of (16) and (17), [45] we obtain: 
Table 1

The optimal values of $\hbar$ for different values of Re and $\mathrm{Ha}$

\begin{tabular}{lcccc}
\hline series solution & $\mathrm{Re}$ & 1.0 & 1.0 & 10.0 \\
\cline { 2 - 5 } & $\mathrm{Ha}$ & 0.0 & 1.0 & 1.0 \\
\hline$V(y)$ & & $\hbar_{\text {Optimal }}=-0.8552$ & $\hbar_{\text {Optimal }}=-0.7163$ & $\hbar_{\text {optimal }}=-0.5434$ \\
$U(y)$ & $\hbar_{\text {Optimal }}=0.3361$ & $\hbar_{\text {Optimal }}=0.4594$ & $\hbar_{\text {Optimal }}=0.1884$ \\
\hline
\end{tabular}

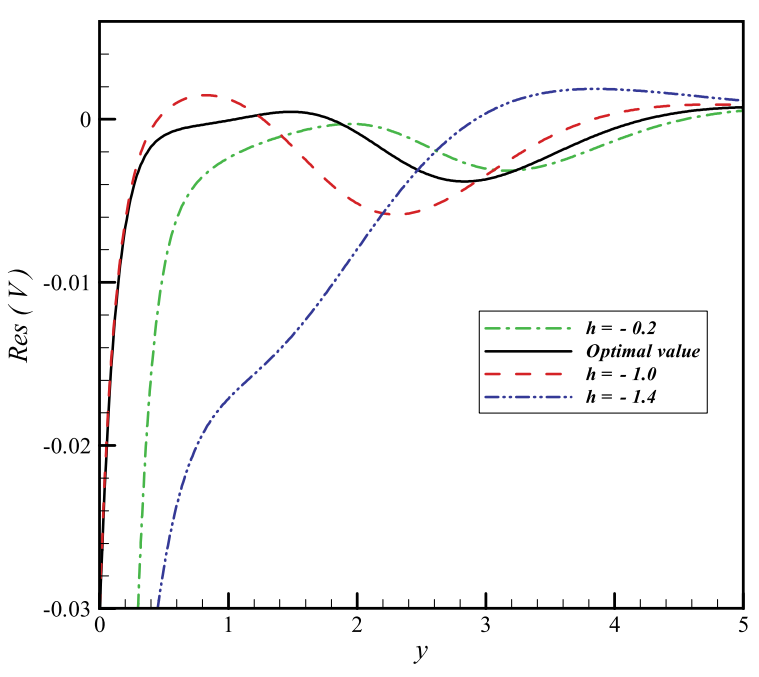

Fig. 4. The behavior of the solutions $\operatorname{Res}_{10}(V)$ obtained by the HAM for various $\hbar$ when $\operatorname{Re}=1.0$ and $H a=1.0$, [45].

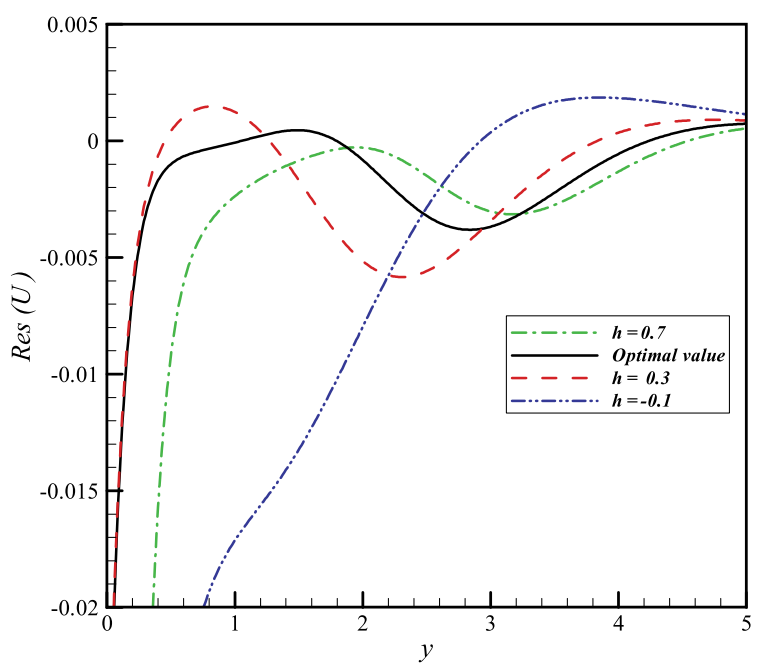

Fig. 5. The behavior of the solutions $\operatorname{Res}_{10}(U)$ obtained by the HAM for various $\hbar$ when $\operatorname{Re}=1.0$ and $H a=1.0$, [45].

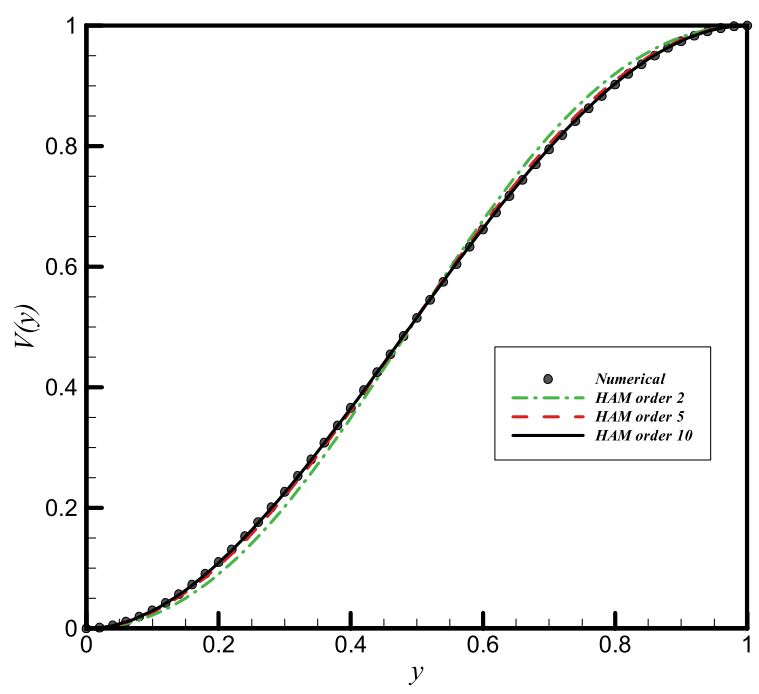

Fig. 6. Comparison of $V(y)$ obtained by various orders of the HAM with numerical solution, when $\operatorname{Re}=1.0$ and $H a=1.0$, [45].

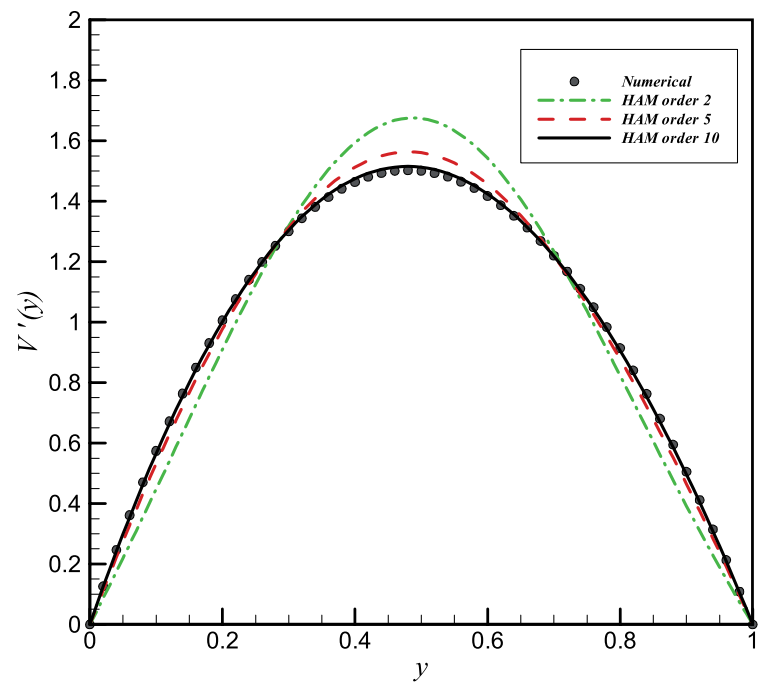

Fig. 7. Comparison of $V^{\prime}(y)$ obtained by various orders of the HAM with numerical solution, when $\mathrm{Re}=1.0$ and $H a=1.0$, [45]. 


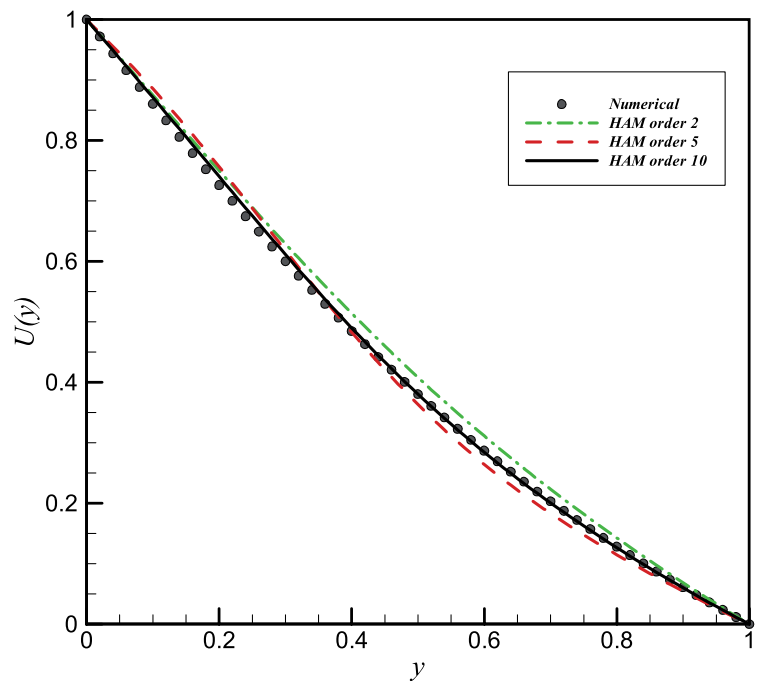

Fig. 8. Comparison of $U(y)$ obtained by various orders of the HAM with numerical solution, when $\mathrm{Re}=1.0$ and $\mathrm{Ha}=1.0$, [45].

$$
\begin{aligned}
& \sum_{k_{1}=0}^{k}\left(k-k_{1}+1\right) G\left(k_{1}\right) F\left(k-k_{1}+1\right) \\
& -\sum_{k_{1}=0}^{k}\left(k-k_{1}+1\right) F\left(k_{1}\right) G\left(k-k_{1}+1\right) \\
& \quad=\frac{1}{\operatorname{Re}}\left((k+1)(k+2) G(k+2)-H a^{2} G(k)\right),
\end{aligned}
$$

$$
\begin{aligned}
& (k+1)(k+2)(k+3)(k+4) F(k+4) \\
& =H a^{2}(k+1)(k+2) F(k+2) \\
& +\operatorname{Re}\left[\sum_{k_{1}=0}^{k}\left(k_{1}+1\right)\left(k-k_{1}+1\right)\right. \\
& \quad\left(k-k_{1}+2\right) F\left(k_{1}+1\right) F\left(k-k_{1}+2\right) \\
& \quad-\sum_{k_{1}=0}^{k}\left(k-k_{1}+1\right)\left(k-k_{1}+2\right) \\
& \left.\left(k-k_{1}+3\right) F\left(k_{1}\right) F\left(k-k_{1}+3\right)\right],
\end{aligned}
$$

where $F(k)$ is the differential transform of $V(y)$ and $G(k)$ is the differential transform of $V(y)$. Transform of the boundary conditions are

$$
\begin{aligned}
& F(0)=0, \quad F(1)=0, \quad F(2)=\alpha, \quad F(3)=\beta, \\
& G(0)=1, \quad G(1)=\gamma,
\end{aligned}
$$

where $\alpha, \beta$ and $\gamma$ are constants. The problem is solved with initial conditions (38) and then the boundary conditions (18) are applied:

$$
\begin{aligned}
& V(1)=1 \text { or } \sum_{k=0}^{N} F(k)=1, \\
& V^{\prime}(1)=0 \text { or } \sum_{k=0}^{N}(k+1) F(k+1)=0, \\
& U(1)=0 \text { or } \sum_{k=0}^{N} G(k)=0 .
\end{aligned}
$$

\section{Entropy generation}

It has been shown [52-54] the volumetric rate of local entropy generation in the presence of magnetic field, can be described as:

$\dot{S}_{g e n}^{\prime \prime \prime}=\frac{k}{T^{2}}[\nabla T]^{2}+\frac{\mu}{T} \Phi+\frac{1}{T}[(J-Q V) .(E+V \times B)]$,

where in a two dimensional Cartesian coordinates we have:

$$
\begin{gathered}
{[\nabla \mathrm{T}]^{2}=\left[\left(\frac{\partial T}{\partial x}\right)^{2}+\left(\frac{\partial T}{\partial y}\right)^{2}\right],} \\
\Phi=2\left[\left(\frac{\partial u}{\partial x}\right)^{2}+\left(\frac{\partial v}{\partial y}\right)^{2}\right]+\left(\frac{\partial u}{\partial y}+\frac{\partial v}{\partial x}\right)^{2}, \\
J=\sigma(E+V \times B) .
\end{gathered}
$$

Since the electric force per unit charge, as compared to $V \times B$ is negligible and also the electric current is much greater than $Q V$, and since the magnetic effect is in $y$-direction only, the Equation (40) can be further simplified as:

$$
\begin{gathered}
\dot{S}_{\text {gen }}^{\prime \prime \prime}=\underbrace{\frac{\mu}{T}\left\{2\left[\left(\frac{\partial u}{\partial x}\right)^{2}+\left(\frac{\partial v}{\partial y}\right)^{2}\right]+\left(\frac{\partial u}{\partial y}+\frac{\partial v}{\partial x}\right)^{2}\right\}}_{\text {Fluid friction irreversibility }} \\
+\underbrace{\frac{\sigma B^{2}}{T}\left(u^{2}\right)}_{\text {Joule dissipation irreversibility }}
\end{gathered}
$$


and based on the velocity component Equation (14), the mentioned derivations also have been obtained as:

$\frac{\partial u}{\partial x}=V^{\prime}, \frac{\partial v}{\partial y}=-V^{\prime}, \frac{\partial u}{\partial y}=u_{0} U^{\prime}+x V^{\prime \prime}, \frac{\partial v}{\partial x}=0$.

So the Equation (44) can be simplified as:

$$
\begin{aligned}
\dot{S}_{\text {gen }}^{\prime \prime \prime}= & \frac{\mu}{T}\left\{2\left[2 V^{\prime 2}\right]+\left(u_{0} U^{\prime}+x V^{\prime \prime}\right)^{2}\right\} \\
& +\frac{\sigma B^{2}}{T}\left(u_{0} U+x V^{\prime}\right)^{2}, \\
\dot{S}_{\text {gen }}^{\prime \prime \prime}= & \frac{\mu}{T}\left\{2\left[2 V^{\prime 2}\right]+\left(u_{0} U^{\prime}+x V^{\prime \prime}\right)^{2}\right\} \\
& +\frac{\mu}{T} \frac{\sigma B^{2} l^{2}}{\mu} \frac{1}{l^{2}}\left(u_{0} U+x V^{\prime}\right)^{2}, \\
\dot{S}_{\text {gen }}^{\prime \prime \prime}= & \frac{\mu}{T}\left(\left\{4 V^{\prime 2}+\left(u_{0} U^{\prime}+x V^{\prime \prime}\right)^{2}\right\}\right. \\
& \left.+H a^{2} \frac{1}{l^{2}}\left(u_{0} U+x V^{\prime}\right)^{2}\right) .
\end{aligned}
$$

By defining the new entropy generation parameter, $N_{G}$, as below we have:

$$
\begin{gathered}
N_{G}=\dot{S}_{\text {gen }}^{\prime \prime \prime} / \frac{\mu}{T}, \\
N_{G}=\left(4 V^{\prime 2}+u_{0}^{2} U^{\prime 2}+x^{2} V^{\prime \prime 2}+u_{0} x U^{\prime} V^{\prime \prime}\right) \\
+H a^{2}\left(\frac{u_{0}^{2} U^{2}}{l^{2}}+\frac{x^{2} V^{\prime 2}}{l^{2}}+\frac{u_{0} x U V^{\prime}}{l^{2}}\right) .
\end{gathered}
$$

\section{Result and discussion}

The governing two-dimensional partial differential equations for problem of steady laminar flow of an electrically conducting fluid in a two dimensional channel are transformed to ordinary differential equations by the Berman similarity transformation. The OHAM and the DTM have been applied successfully to derive the semi-numerical solutions under appropriate boundary conditions. Then the entropy generation parametric analysis has been done.

Firstly, for the comparisons between 10th-order approximation of the OHAM and 20th-order approximation of the DTM, also the physical interpretation of the magneto-hemodynamics studied in the present problem, the OHAM solutions for the effects of suction Reynolds number and Hartmann number are presented

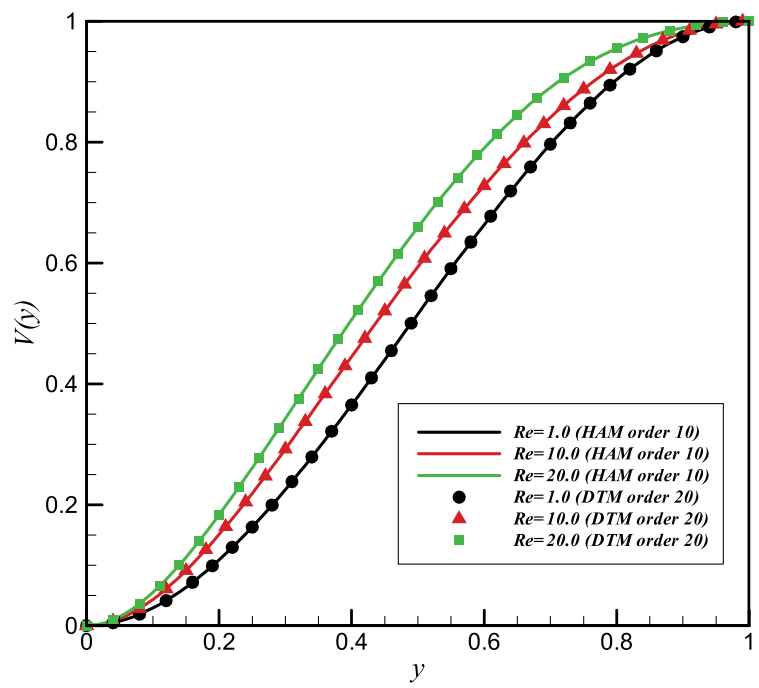

Fig. 9. The profile $V(y)$ obtained by the 10th-order approximation of the HAM and 20th-order approximation of the DTM for different values of $\mathrm{Re}$, when $\mathrm{Ha}=0.0$, [45].

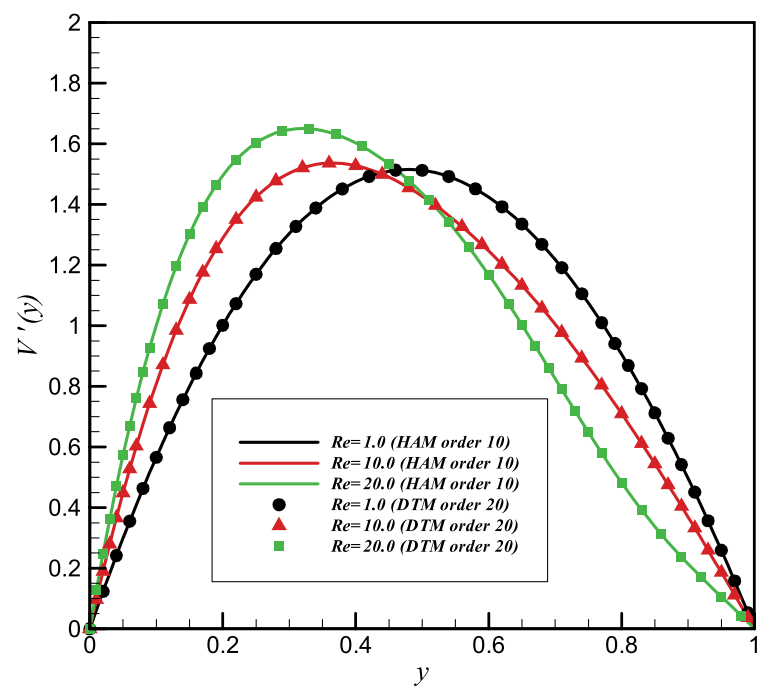

Fig. 10. The profile $V^{\prime}(y)$ obtained by the 10 th-order approximation of the HAM and 20th-order approximation of the DTM for different values of $\mathrm{Re}$, when $\mathrm{Ha}=0.0$, [45].

in Figs. 9-11 and 12-14, respectively. Also the numerical comparisons between 10th-order approximation of the OHAM, 20th-order approximation of the DTM and the numerical quadrature solutions for $\operatorname{Re}=1.0$ and $H a=0.0$ are tabulated in the Tables 2-4 [45].

Figure 9 illustrates the evolution of fluid velocity profiles across the channel width $(0 \leq \mathrm{y} \leq 1)$ for $H a=0.0$ (Lorentzian hydromagnetic drag and viscous 


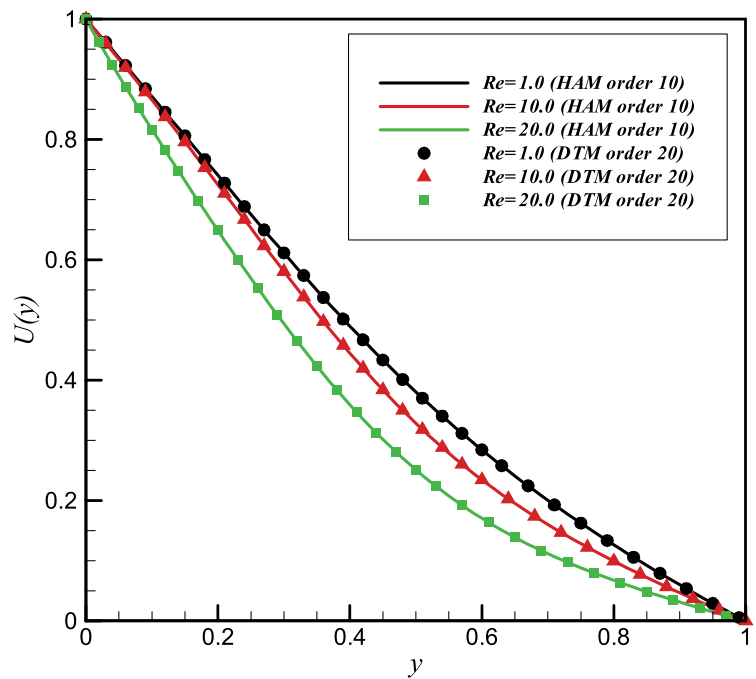

Fig. 11. The profile $U(y)$ obtained by the 10 th-order approximation of the HAM and 20th-order approximation of the DTM for different values of $\mathrm{Re}$, when $\mathrm{Ha}=0.0$, [45].

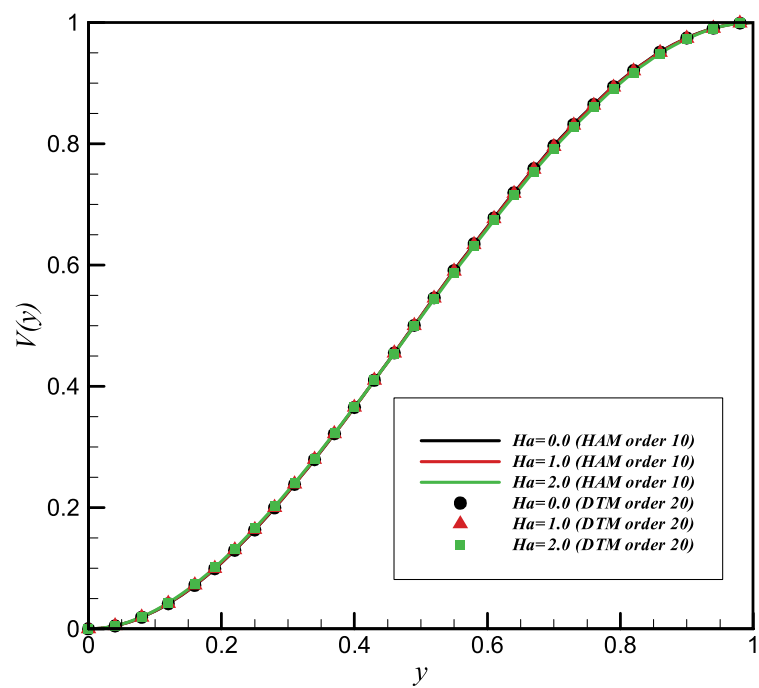

Fig. 12. The profile $V(y)$ obtained by the 10 th-order approximation of the HAM and 20th-order approximation of the DTM for different values of $H a$, when $\mathrm{Re}=1.0$, [45].

force in the flow are equal and the flow is therefore weakly magnetic) obtained by OHAM and DTM. As Re increase suction at the upper plate increase. Increasing lateral mass flux out of the upper plate will drag the fluid towards the upper plate. This will serve to enhance momentum in the $y$-direction and deplete momentum in the $x$-direction. As a result the $V(y)$ component of blood velocity will be enhanced, as shown in Fig. 9.

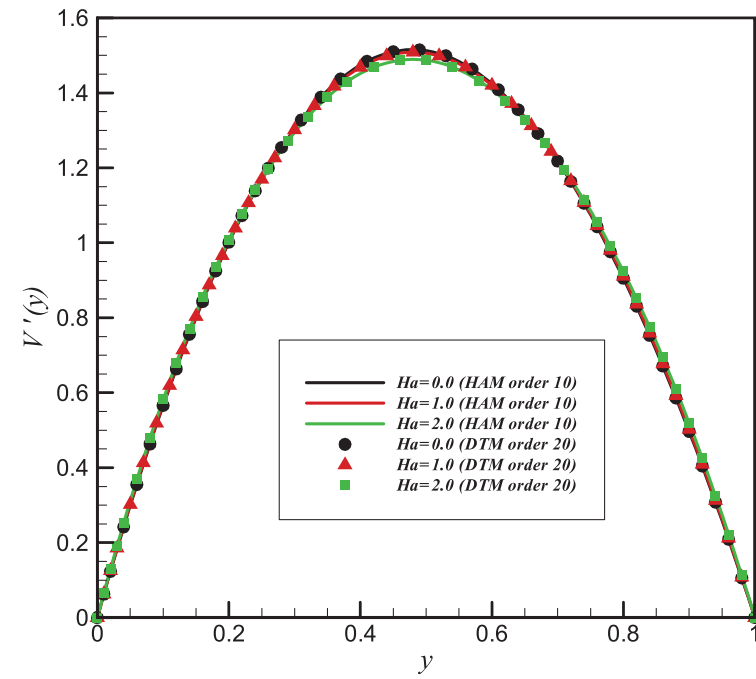

Fig. 13. The profile $V^{\prime}(y)$ obtained by the 10th-order approximation of the HAM and 20th-order approximation of the DTM for different values of $H a$, when $\mathrm{Re}=1.0$, [45].

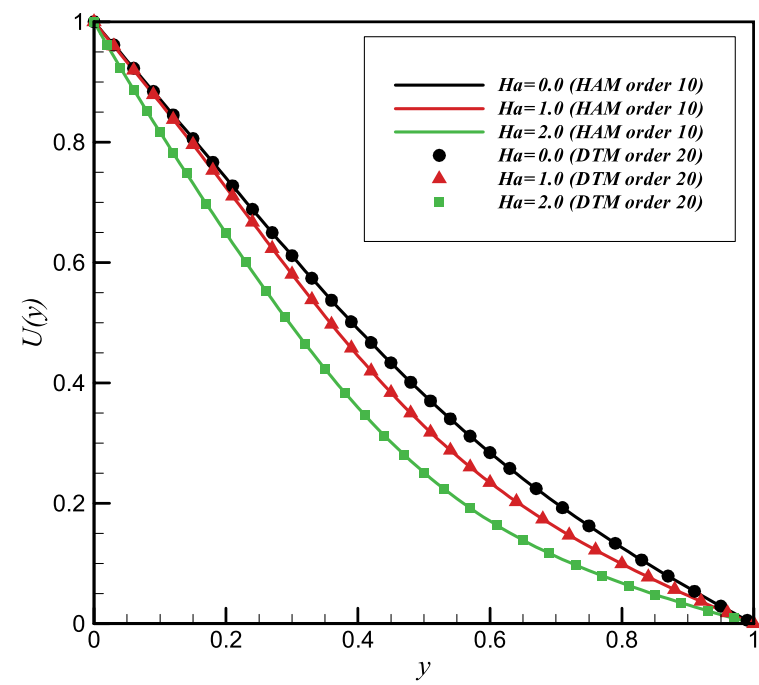

Fig. 14. The profile $U(y)$ obtained by the 10th-order approximation of the HAM and 20th-order approximation of the DTM for different values of $H a$, when $\operatorname{Re}=1.0$, [45].

Also it is observed that results of the OHAM are very close to the DTM which confirm the validity of these methods. Similarly velocity gradient, $V^{\prime}(y)$ i.e. dimensionless transverse shear stress component, will be boosted in the channel initially, (Fig. 10) as testified by the first half of the plots, where for $\mathrm{Re}=20.0$, velocity gradient is maximized. This trend is however reversed as we approach the upper channel, since the 


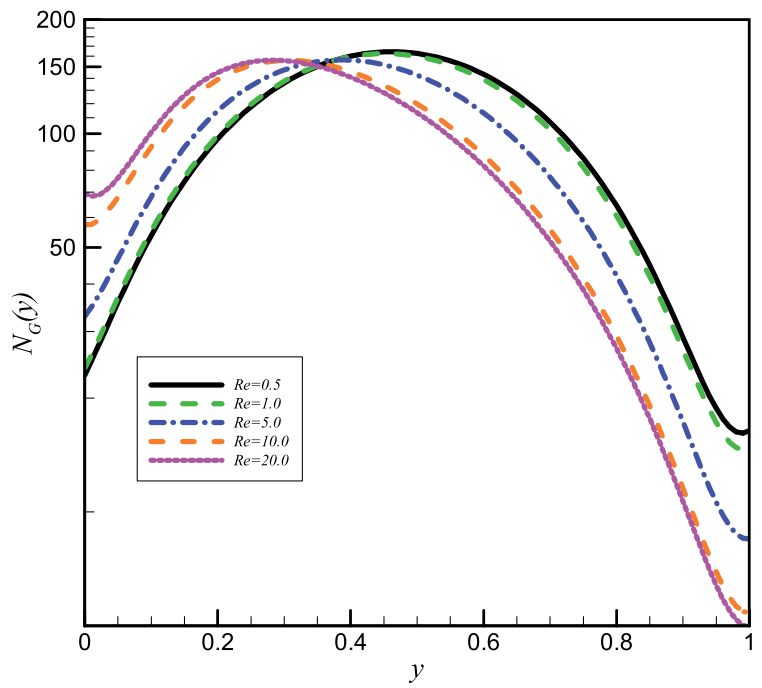

Fig. 15. Variation of entropy generation parameter, $N_{G}(y)$, with respect to $y$ for different values of Reynolds number.

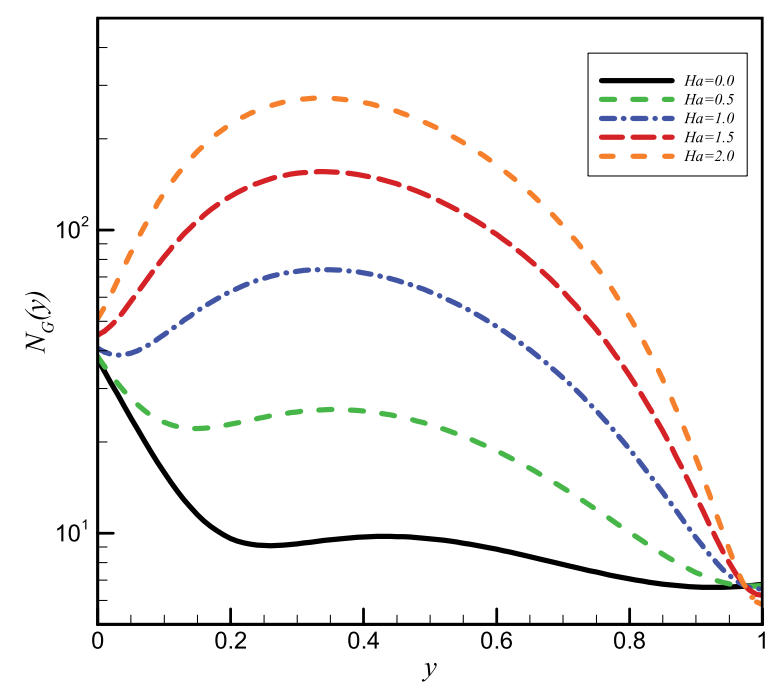

Fig. 16. Variation of entropy generation parameter, $N_{G}(y)$, with respect to $y$ for different values of Hartmann number.

imposition of a suction effect here, while boosting the velocity, can simultaneously depress the rate of change of velocity, as indicated by Skalak and Wang [55] and also by Quaile and Levy [56] for the non-conducting cases $(H a=0.0)$.

Figure 11 shows that increasing suction Reynolds number values from 1 through 10 to 20 , lead to a strong deceleration in the $\mathrm{x}$-direction velocity, $U(y)$. Evans and Skalak [57] also identified this trend and explained that in oxygen diffusion processes.

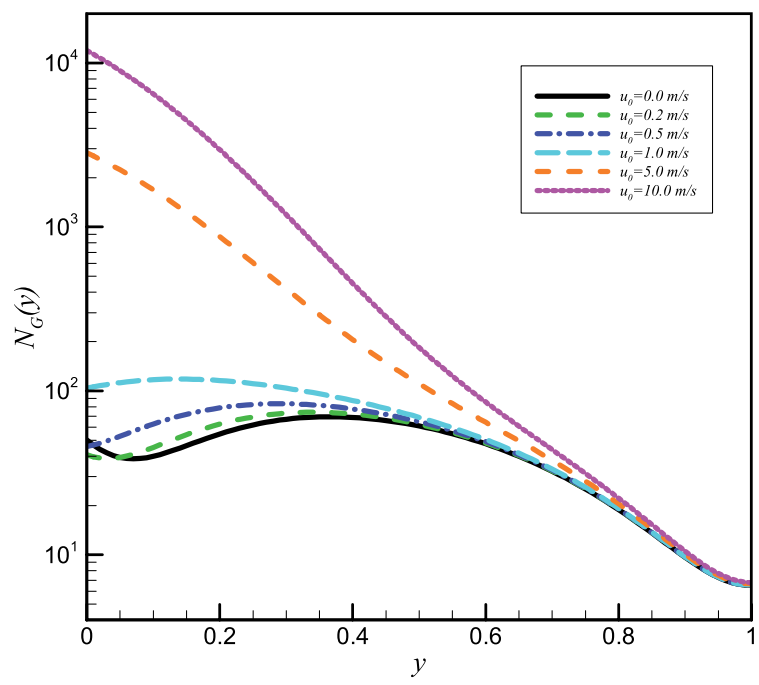

Fig. 17. Variation of entropy generation parameter, $N_{G}(y)$, with respect to $y$ for different values of $x$-velocity of the moving plate.

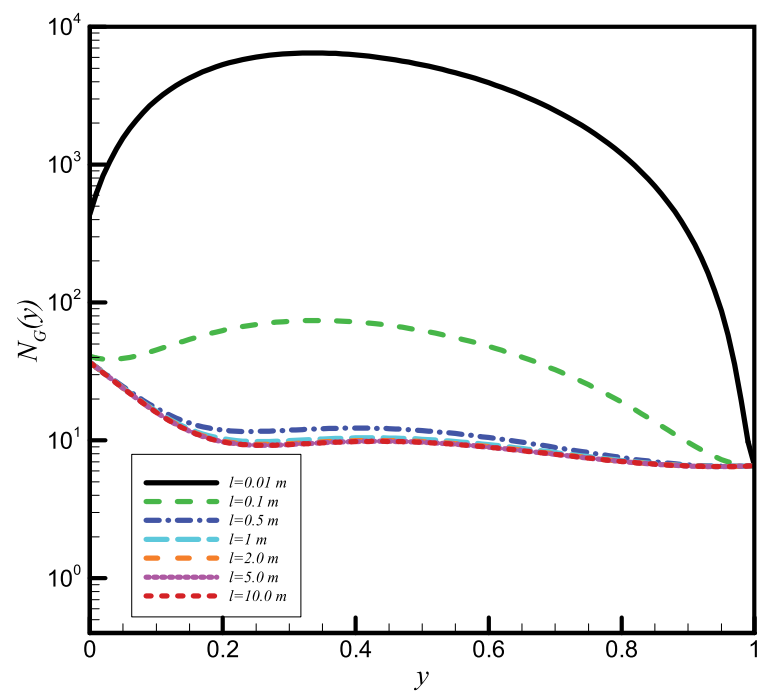

Fig. 18. Variation of entropy generation parameter, $N_{G}(y)$, with respect to $y$ for different values of suspension height.

Figure 12 shows the y-direction velocity, $V(y)$ for $\mathrm{Re}=1.0$ and various quantity of $\mathrm{Ha}$ obtained by OHAM and DTM. It should be noted that as the magnetic field is applied transverse to the channel length, the Lorentz force component generated will be perpendicular to the y-direction. This implies that magnetohydrodynamic drag will not substantially influence the transverse velocity distribution, $V(y)$, and will serve to principally influence the longitudinal flow, $U(y)$. With a rise in $\mathrm{Ha}$ from 0 (non-conducting 


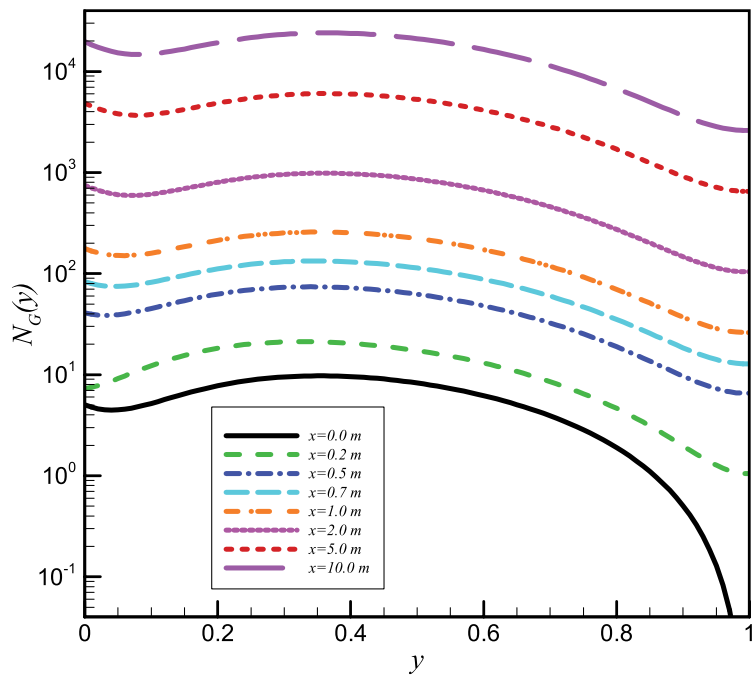

Fig. 19. Variation of entropy generation parameter, $N_{G}(y)$, with respect to $y$ for different values of dimensionless horizontal coordinate.

Table 2

Comparison of values of $V(y)$ obtained by various orders of the $\mathrm{HAM}$ with numerical solution, when $\mathrm{Re}=1.0$ and $\mathrm{Ha}=1.0$

\begin{tabular}{lccl}
\hline$y$ & \multicolumn{3}{c}{$V(y)$} \\
\cline { 2 - 4 } & HAM order 10 & DTM order 20 & Numerical \\
\hline 0.0 & 0 & 0 & 0 \\
0.1 & 0.0296593 & 0.0296302 & 0.029608 \\
0.2 & 0.109451 & 0.109735 & 0.10944 \\
0.3 & 0.225821 & 0.227135 & 0.225922 \\
0.4 & 0.365433 & 0.368652 & 0.365612 \\
0.5 & 0.515204 & 0.521106 & 0.515428 \\
0.6 & 0.662495 & 0.671318 & 0.662796 \\
0.7 & 0.795347 & 0.80611 & 0.795761 \\
0.8 & 0.902645 & 0.912303 & 0.90308 \\
0.9 & 0.974092 & 0.976717 & 0.974323 \\
1.0 & 1 & 0.986173 & 1 \\
\hline
\end{tabular}

Table 3

Comparison of values of $V^{\prime}(y)$ obtained by various orders of the $\mathrm{HAM}$ with numerical solution, when $\mathrm{Re}=1.0$ and $\mathrm{Ha}=1.0$

\begin{tabular}{lccc}
\hline$y$ & \multicolumn{3}{c}{$V^{\prime}(y)$} \\
\cline { 2 - 4 } & HAM order 10 & DTM order 20 & Numerical \\
\hline 0.0 & 0 & 0. & 0 \\
0.1 & 0.570067 & 0.57064 & 0.569789 \\
0.2 & 1.0032 & 1.00549 & 1.00417 \\
0.3 & 1.30196 & 1.30655 & 1.30303 \\
0.4 & 1.46843 & 1.46982 & 1.46895 \\
0.5 & 1.50589 & 1.50733 & 1.50639 \\
0.6 & 1.41997 & 1.42699 & 1.42103 \\
0.7 & 1.21855 & 1.22689 & 1.21952 \\
0.8 & 0.910285 & 0.91097 & 0.909457 \\
0.9 & 0.502562 & 0.503716 & 0.499552 \\
1.0 & 0 & 0 & 0 \\
\hline
\end{tabular}

Table 4

Comparison of values of $U(y)$ obtained by various orders of the HAM with numerical solution, when $\mathrm{Re}=1.0$ and $\mathrm{Ha}=1.0$

\begin{tabular}{lccc}
\hline$y$ & \multicolumn{3}{c}{$U(y)$} \\
\cline { 2 - 4 } & HAM order 10 & DTM order 20 & Numerical \\
\hline 0.0 & 1 & 1 & 1 \\
0.1 & 0.85134 & 0.839368 & 0.839368 \\
0.2 & 0.708228 & 0.692234 & 0.692213 \\
0.3 & 0.573 & 0.560475 & 0.560339 \\
0.4 & 0.450177 & 0.444584 & 0.444081 \\
0.5 & 0.343064 & 0.34404 & 0.342701 \\
0.6 & 0.252133 & 0.257632 & 0.254742 \\
0.7 & 0.175302 & 0.183744 & 0.178368 \\
0.8 & 0.109373 & 0.120592 & 0.111631 \\
0.9 & 0.0515228 & 0.0664037 & 0.0526971 \\
1.0 & 0 & 0 & 0 \\
\hline
\end{tabular}

case) through 1 to 2.0 , there is no tangible variation in the velocity component, $V(y)$. According to Fig. 13, a slight reduction in velocity gradient, $V^{\prime}(y)$ is observed. In Fig. 14 a substantial deceleration in longitudinal velocity $U(y)$, is induced with increasing $\mathrm{Ha}$ through the depth of the channel. Clearly further increase in $\mathrm{Ha}$ and $\mathrm{Re}$ will further induce a stronger retardation in the longitudinal flow and the present computations demonstrate that by micro-machining the upper plate properly and imposing stronger magnetic fields, biomedical engineering surgeons can achieve even greater control of blood flow during operations. Also from Figs 9-14, it is observed that results of the OHAM are very close to the DTM which confirm the validity of these methods.

The importance of this study is entropy generation parametric analysis. So second, effect of all different mentioned effective parameters on the entropy generation has been analyzed. In all of these figures constant parameters, except which ones that highlighted in each figure, have the values as $\mathrm{Re}=10, \mathrm{Ha}=1, \mathrm{u}_{0}=$ $0.2, l=0.1, x=0.5$.

At first the effect of Reynolds number on $N_{G}(y)$ has been presented in Fig. 15. As can be seen in this figure for all values of $\operatorname{Re}, N_{G}(y)$ has the same trend respect to increasing in $y$, it increases to a maximum value and then decreases. Along with the mentioned maximum value, $N_{G}(y)$ has its minimum value at $y=1$. As can be seen in this figure, by increasing in Re, the maximum value of $N_{G}(y)$ is nearly constant but the position of these maximums are in the smaller values of $y$. Besides, the minimum values at $y=1$ decreases with increasing in Re. Figure 16 shows the effect of Hartmann number on $N_{G}(y)$. As can be seen in this figure by increasing in Ha the curves of $N_{G}(y)$ are quite similar except for $H a=0$. It is clear that the bound- 
ary values of $N_{G}(y)$, it means $N_{G}(y)$ at $y=0$ and 1 , are independent from the variation of Ha and the only effect of increasing in $\mathrm{Ha}$ on $N_{G}(y)$ is the increasing in its maximum values. Figure 17 presents the effect of changing in $x$-velocity of the moving plate on $N_{G}(y)$. The maximum vales of $N_{G}(y)$ are at $y=0$ for the cases with about $u_{0}>1.0 \mathrm{~m} / \mathrm{s}$, whereas for $u_{0}<1.0 \mathrm{~m} / \mathrm{s}$ the maximum values are in $y>0$ with different values for each case. As another study, the effect of suspension height on the $N_{G}(y)$ has been illustrated in Fig. 18 . As can be seen this parameter's effect is noticeable at $l<0.5 \mathrm{~m}$. Finally the effect of dimensionless horizontal coordinate has been analyzed as presented in Fig. 19. It can be seen that by increasing in $x$ all the curves are same nearly and they shift to the higher values only. It means that the trend of these curves is the same but their values change.

\section{Conclusions}

In this paper, as the prerequisite of the entropy generation study, the problem of laminar viscous magneto-hemodynamic flow in a semi-porous channel under a transverse magnetic field has been solved using two powerful techniques, namely the optimal homotopy analysis method (OHAM) and the differential transform method (DTM). The importance of this paper is parametric analysis of entropy generation of this problem. After finding the solutions of OHAM and DTM, entropy generation of this system has been analyzed and effects of the Reynolds number, Hartmann number, $x$-velocity of the moving plate, suspension height and dimensionless horizontal coordinate on the entropy generation have been studied. The importance of this study is its use for minimizing entropy generation in the industrial applications. The present analyses have provided some further insights into the thermodynamics of proposed problem, because the main goal of this research is to consider this important thermodynamics phenomenon in calculations of such systems.

\section{Acknowledgments}

O. Anwar Bég and Tasveer A. Bég wish to acknowledge the tremendous inspiration provided to them by their late mother, Javida K. Bég (1946-2010), she had the most profound and positive influence on their careers in engineering science research.

\section{References}

[1] A. Ishak, R. Nazar and I. Pop, MHD boundary-layer flow of a micropolar fluid past a wedge with constant wall heat flux, Communications in Nonlinear Science and Numerical Simulation 14 (2009), 109-118.

[2] A.B. Parsa, M.M. Rashidi and T. Hayat, MHD boundary-layer flow over a stretching surface with internal heat generation or absorption, Heat Transfer-Asian Research 42(6) (2013), 500-514.

[3] T. Hayat, C. Fetecau and M. Sajid, Analytic solution for MHD Transient rotating flow of a second grade fluid in a porous space, Nonlinear Analysis: Real World Applications 9 (2008), 1619-1627.

[4] T. Hayat, T. Javedb and M. Sajid, Analytic solution for MHD rotating flow of a second grade fluid over a shrinking surface, Physics Letters A 372 (2008), 3264-3273.

[5] S. Ichioka, S.M. Minegishi, M. Iwasaka, M. Shibata, T. Nakatsuka, K. Harii, A. Kamiya and S. Ueno, High-intensity static magnetic fields modulate skin microcirculation and temperature in vivo, Bioelectromagnetics 21 (2000), 183-188.

[6] T. Higashi, A. Yamagishi, T. Takeuchi, N. Kawaguchi, S. Sagawa, S. Onishi and M. Date, Orientation of erythrocytes in a strong static magnetic field, J Blood 82 (1993), 1328-1334.

[7] T.S. Tenforde, Magnetically induced electric fields and currents in the circulatory system, Prog Biophys Mol Biol 87 (2005), 279-288.

[8] Y. Haik, V. Pai and C.J. Chen, Apparent viscosity of human blood in high static magnetic field, J Magnetism and Magnetic Materials 225 (2001), 180-186.

[9] O.A. Bég, Giants of Engineering Science, 110 pp, Matador, UK (2003). (Chinese Translation, January 2008).

[10] V.R. Prasad, B. Vasu and O.A. Bég, Thermo-Diffusion and Diffusion-Thermo Effects on Boundary Layer Flows, Lambert Academic Publishing, Germany, 220 pages, 978-38443-9745-1, May (2011).

[11] J.R. Keltner, M.S. Roos, P.R. Brakeman and T.F. Budinger, Magneto hydrodynamics of blood flow, Mag Reson Med J 16 (1990), 139-149.

[12] V.K. Sud and G.S. Sekhon, Blood flow through the human arterial system in the presence of a steady magnetic field, Biophysical J 84 (2003), 2638-2645.

[13] A.R. Rao and K.S. Desikachar, Diffusion in hydromagnetic oscillatory flow through a porous channel, ASME J Applied Mechanics 54 (1987), 742-753.

[14] N. Mustapha, N. Amin, S. Chakravarty and P.K. Mandal, Unsteady magnetohydrodynamic blood flow through irregular multi-stenosed arteries, Computers in Biology and Medicine 39 (2009), 896-906.

[15] F. Paul, S. Roath and D. Melville, Differential blood cell separation using a high gradient magnetic field, British $J$ Haematology 38 (1978), 273-280.

[16] G. Ramamurthy and B. Shanker, Magnetohydrodynamic effects on blood flow through a porous channel, Medical and Biological Engineering and Computing 32 (1994), 655-659.

[17] H.S. Takhar, R. Bhargava, S. Rawat, T.A. Bég, O.A. Bég and T.K. Hung, Biomagnetic hydrodynamics in a 2-dimensional non-Darcian porous medium: Finite element study, $J$ Theoretical and Applied Mechanics 37(2) (2007), 59-76.

[18] R. Bhargava, S. Rawat, H.S. Takhar and O.A. Bég, Pulsatile magneto-biofluid flow and mass transfer in a non-Darcian porous medium channel, Mecannica 42 (2007), 247-262. 
[19] S.K. Pandey and D. Tripathi, Influence of magnetic field on peristaltic flow of viscous fluid through a finite length cylindrical tube, Applied Bionics and Biomechanics 7 (2010), 169-176.

[20] K.S. Mekheimer, Peristaltic flow of blood under effect of a magnetic field in a non-uniform channel, Applied Mathematics and Computation 153(3) (2004), 763-777.

[21] S.K. Pandey and D. Tripathi, Peristaltic flow characteristics of Maxwell and magneto-hydrodynamic fluids in finite channels: Models for oesophageal swallowing, J Biological Systems 18 (2010), 10-14.

[22] W. Kainz et al., Development and validation of a magnetohydrodynamic solver for blood flow analysis, Phys Med Biol 55 (2010), 7253-7273.

[23] K. Das and G.C. Saha, Mathematical analysis on MHD pulsatile flow of blood through a rough thin-walled elastic tube, Applied Mathematical Sciences 4 (2010), 2463-2473.

[24] Kh. S. Mekheimer and M.A. El Kot, Effect of lateral walls on peristaltic flow through an asymmetric rectangular duct, Applied Bionics and Biomechanics 8 (2011), 1-14.

[25] O. Wernert, H. Schäf and R. Ghobarkar, Denoyel, Adsorption properties of zeolites for artificial kidney applications, Microporous and Mesoporous Materials 83 (2005), 101-113.

[26] A. Jafari, P. Zamankhan, S.M. Mousavi and P. Kolari, Numerical investigation of blood flow Part II: In capillaries, Communications in Nonlinear Science and Numerical Simulation 14 (2009), 1396-1402.

[27] A.R. Goerke, J. Leung and S.R. Wickramasinghe, Mass and momentum transfer in blood oxygenators, Chemical Engineering Science 57 (2002), 2035-2046.

[28] A.S. Berman, Laminar flow in channels with porous walls, $J$ Appl Phys 24 (1953), 1232-1235.

[29] A. Desseaux, Influence of a magnetic field over a laminar viscous flow in a semi-porous channel, Int J Engineering Science 37 (1999), 94-102.

[30] Kh. S. Mekheimer, S.Z.A. Husseny and A.I. Abd el Lateef, Suspension model for blood flow through arterial catheterization, Chemical Eng Communication J 197(9) (2010), 1195-1214.

[31] A.H. Nayfeh, Perturbation methods, New York, Wiley, 2000.

[32] E. Erfani, M.M. Rashidi and A. Basiriparsa, The modified Differential Transform Method for solving off-centered stagnation flow towards a rotating disc, International Journal of Computational Methods 7(4) (2010), 655-670.

[33] M.M. Rashidi, N. Laraqi and A. BasiriParsa, Analytical modeling of heat convection in magnetized micropolar fluid by using modified differential transform method, Heat TransferAsian Research 40(3) (2011), 187-204.

[34] S.J. Liao, The proposed homotopy analysis technique for the solution of nonlinear problems, $\mathrm{PhD}$ thesis, Shanghai Jiao Tong University, 1992.

[35] M.M. Rashidi, G. Domairry and S. Dinarvand, Approximate solutions for the Burger and regularized long wave equations by means of the homotopy analysis method, Communications in Nonlinear Science and Numerical Simulation 14 (2009), 708-717.

[36] Z. Ziabakhsh and G. Domairry, Analytic solution of natural convection flow of a non-Newtonian fluid between two vertical flat plates using homotopy analysis method, Commun Non-linear Sci Numer Simulat 14 (2009), 1868-1880.

[37] M.M. Rashidi, S.A. Mohimanian Pour, T. Hayat and S. Obaidat, Analytic approximate solutions for steady flow over a rotating disk in porous medium with heat transfer by homotopy analysis method, Computers \& Fluids 54 (2012), 1-9.

[38] M. Sajid, T. Hayat and S. Asghar, Comparison between the HAM and HPM solutions of thin film flows of non-Newtonian fluids on a moving belt, Nonlinear Dynam 50 (2007), 27-35.

[39] J.K. Zhou, Differential Transformation and Its Applications for Electrical Circuits, Huazhong University Press, Wuhan, China, 1986 (in Chinese).

[40] C.K. Chen and S.H. Ho, Solving partial differential equations by two dimensional differential transform method, Applied Mathematics and Computation 106 (1999), 171-179.

[41] F. Ayaz, Solutions of the systems of differential equations by differential transform method, Applied Mathematics and Computation 147 (2004), 547-567.

[42] M.J. Jang, C.L. Chen and Y.C. Liu, Two-dimensional differential transform for partial differential equations, Applied Mathematics and Computation 121 (2001), 261-270.

[43] A. Kurnaz, G. Oturnaz and M.E. Kiris, N-Dimensional differential transformation method for solving linear and nonlinear PDE's, Int J Computational Mathematics 82 (2005), 369-380.

[44] M.M. Rashidi, O. Anwar Bég, M. Asadi and M.T. Rastegari, DTM-Padé modeling of natural convective boundary layer flow of a nanofluid past a vertical surface, Int $J$ Thermal and Environmental Engineering 4 (2012), 13-24.

[45] A.B. Parsa, M.M. Rashidi, O. Anwar Bég and S.M. Sadri, Semi-computational simulation of magneto-hemodynamic flow in a semi-porous channel using optimal homotopy and differential transform methods, Computers in Biology and Medicine 43(9) (2013), 1142-1153.

[46] A. Bejan, in: P.H. James, F.I. Thomas (Eds.), Advances in Heat Transfer, (1982).

[47] S. Aiboud and S. Saouli, Entropy analysis for viscoelastic magneto hydrodynamic flow over a stretching surface, Int $J$ Non Linear Mech 45(5) (2010), 482-489.

[48] M.M. Rashidi, S. Abelman and N. FreidooniMehr, Entropy generation in steady MHD flow due to a rotating porous disk in a nanofluid, International Journal of Heat and Mass Transfer 62 (2013), 515-525

[49] M.M. Rashidi, L. Shamekhi and S. Kumar, Parametric Analysis of Entropy Generation in Off-centered Stagnation Flow Towards a Rotating Disc, Nonlinear Engineering, In press.

[50] J.F. Osterle and F.J. Young, Natural convection between heated vertical plates in a horizontal magnetic field, $J$ Fluid Mechanics 11 (1961), 512-518.

[51] J.C. Umavathi, A note on magnetoconvection in a vertical enclosure, Int J Non-Linear Mechanics 31 (1996), 371-376.

[52] A. Bejan, Entropy generation minimization, the method of thermodynamic optimization of finite-size systems and finitetime processes, CRC Press, (1996).

[53] A. Arikoglu, I. Ozkol and G. Komurgoz, Effect of slip on entropy generation in a single rotating disk in MHD flow, Applied Energy 85(12) (2008), 1225-1236.

[54] A. Bejan, Entropy generation through heat and fluid flow, Wiley, (1982).

[55] F.M. Skalak And and C.Y. Wang, On the non-unique solutions of laminar flow through a porous tube or channel, SIAM J Appl Math 34 (1978), 535-544.

[56] J.P. Quaile and E.K. Levy, Laminar flow in a porous tube with suction, Int J Heat Mass Transfer 97 (1975), 223-243.

[57] E.A. Evans and R. Skalak, Mechanics and Thermodynamics of Biomembranes, CRC Press, Florida, USA, (1980). 

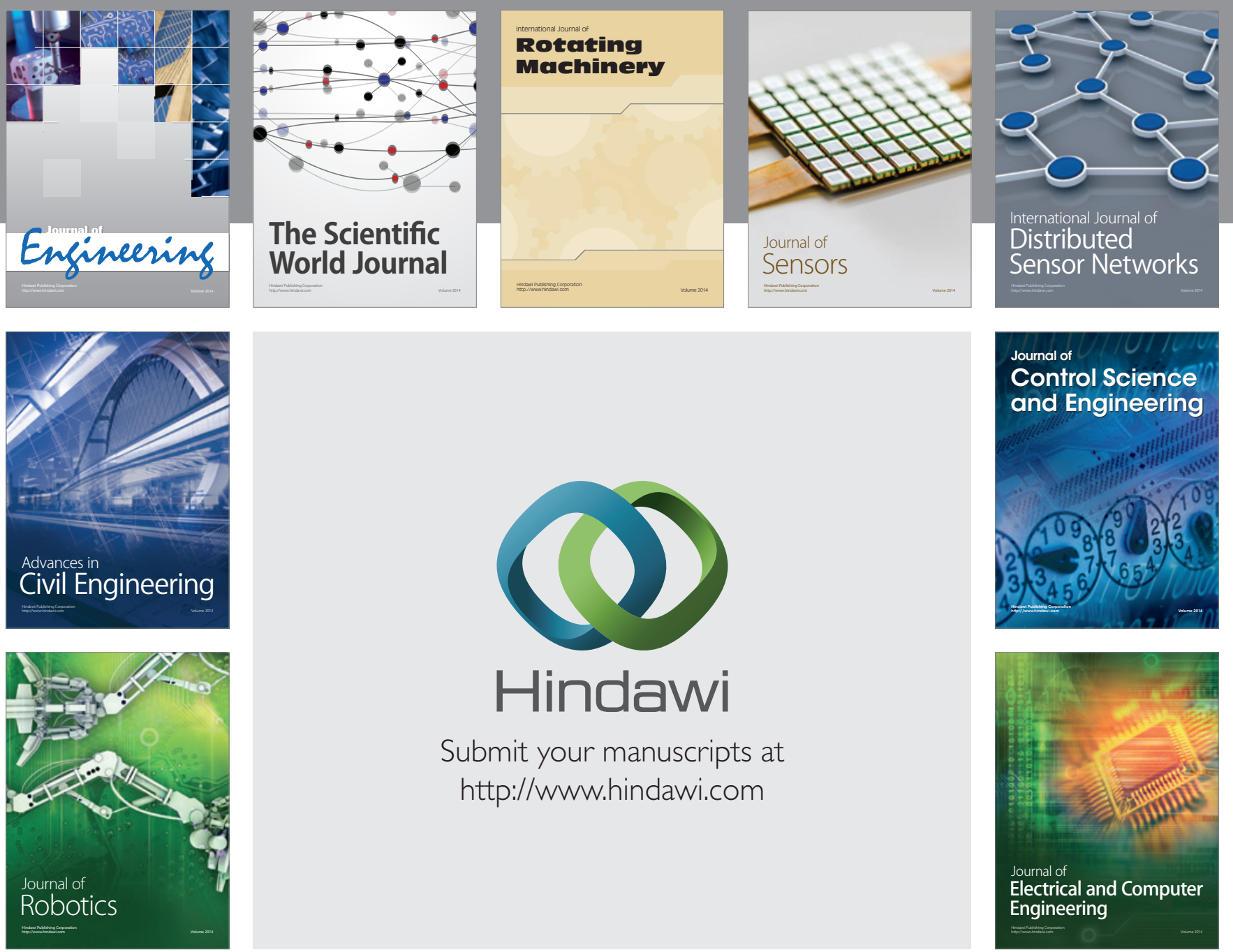

Submit your manuscripts at

http://www.hindawi.com
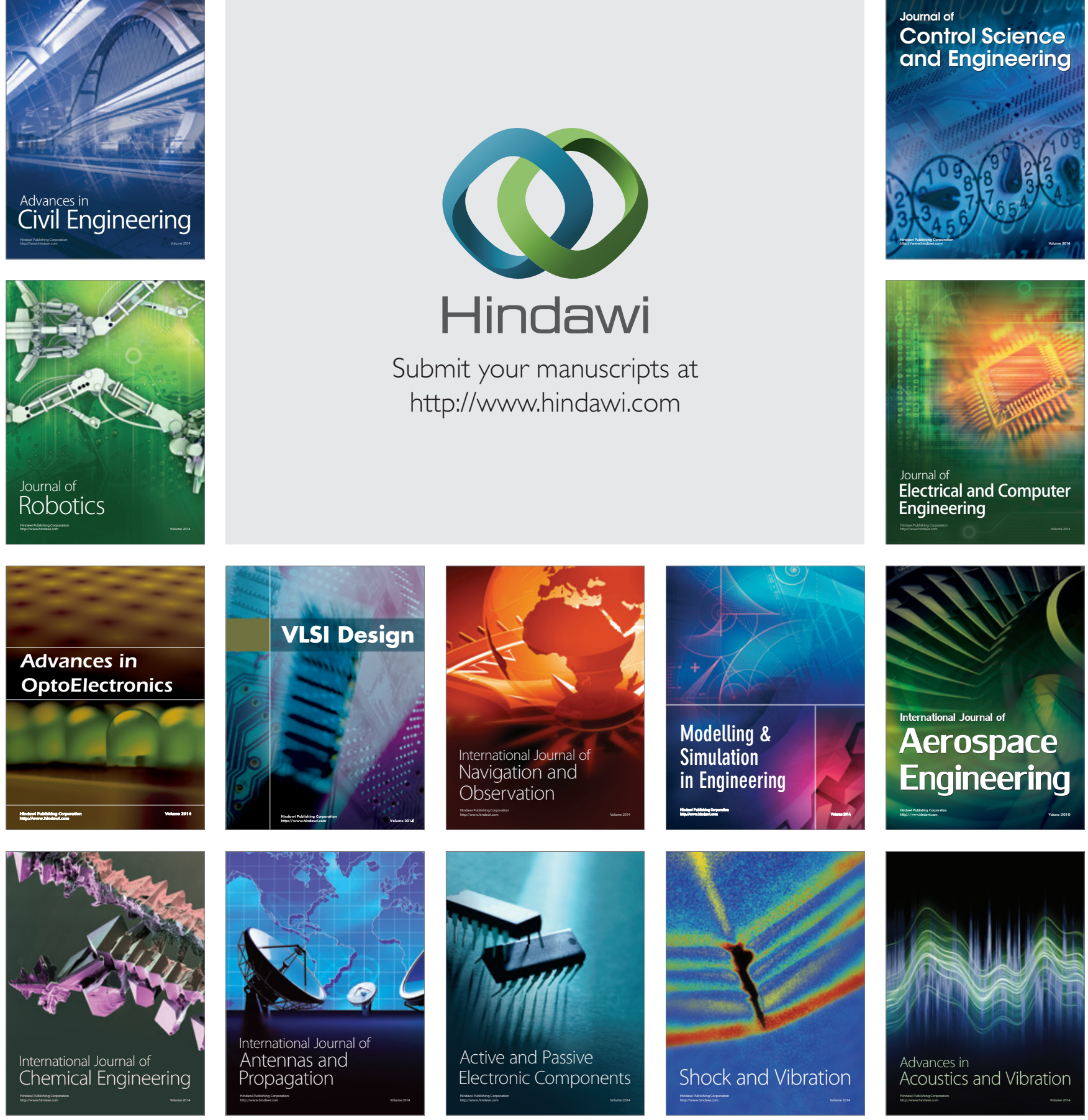